\title{
Capacity Analysis of Distributed Antenna Systems with Multiple Receive Antennas over MIMO Fading Channel
}

\author{
Xiangbin Yu, ${ }^{1,2}$ Xiaoshuai Liu, ${ }^{1}$ Yuyu Xin, ${ }^{1}$ Ming Chen, ${ }^{2}$ and Yun Rui ${ }^{3}$ \\ ${ }^{1}$ Department of Electronic Engineering, Nanjing University of Aeronautics and Astronautics, Nanjing 210016, China \\ ${ }^{2}$ National Mobile Communications Research Laboratory, Southeast University, Nanjing 210096, China \\ ${ }^{3}$ Shanghai Advanced Research Institute, Chinese Academic of Science, Shanghai 201203, China
}

Correspondence should be addressed to Xiangbin Yu, xiyangyang2004@163.com

Received 8 November 2011; Revised 19 January 2012; Accepted 24 January 2012

Academic Editor: Charles Bunting

Copyright () 2012 Xiangbin Yu et al. This is an open access article distributed under the Creative Commons Attribution License, which permits unrestricted use, distribution, and reproduction in any medium, provided the original work is properly cited.

\begin{abstract}
The downlink performance and capacity of distributed antenna systems (DASs) with multiple receive antennas are investigated in multi-input multi-output (MIMO) fading and multicell environment. Based on the moment generating function and performance analysis, an exact closed-form expression of DAS ergodic capacity is derived, and it includes the existing capacity expression as a special case. Moreover, a simple closed-form approximate expression of ergodic capacity is also derived by using the Taylor series, and it has the performance result close to the exact expression. Besides, the outage capacity of DAS is analyzed, and an exact closed-form expression of outage capacity probability is derived. All these expressions can provide good theoretical performance evaluation for DAS. Simulation results corroborate our theoretical analysis.
\end{abstract}

\section{Introduction}

As a promising technique for future wireless communications, the distributed antenna system has received considerable attention in recent years. It can significantly improve the system capacity and cell coverage and reduce power consumption in comparison with traditional centralized antenna systems, and thus it has become one of the key technologies in next generation mobile communication systems such as 3G-LTE (long term evolution) and IMTadvanced [1-3]. Based on different system models, the capacity performance of distributed antenna systems (DAS) has been extensively studied in [4-9] and references therein. The effect of maximal-ratio-combining- (MRC-) based macrodiversity on the outage capacity in code division multiple access (CDMA) DAS is analyzed in [4]. The uplink capacity in the generalized DAS environment is studied in [5], and closed-form expressions of ergodic capacity are derived by using two approximation distributions, but the performance analysis is limited in single cell. The downlink capacity of DAS in multicell environment is analyzed in [6], and a closed-form expression of ergodic capacity is derived. Considering different cooperation strategies, the capacity performance of DAS under the downlink is investigated in [7], but the influence of noise is neglected. The downlink capacity of DAS in multiuser scenario is provided in [8], but the performance analysis is limited in single cell with three distributed antennas for analysis simplicity. The uplink and downlink capacity of DAS over shadowed fading channels are, respectively, studied in our previous papers $[3,9]$, and approximate closed-form capacity expressions are derived for single-cell case.

Although, in all these studies, the DAS capacity is well analyzed, only limited work has been carried out for DAS in the multicell environment. Especially, the capacity analysis of DAS with multiple antennas at the mobile station (MS) for multicell environment is not provided in the existing literature. Motivated by the above reasons, in this paper, we will address the downlink capacity study of DAS with multiple receive antennas in the multicell environment and focus on the derivation of accurate capacity expressions of DAS in multi-input multi-output (MIMO) Rayleigh fading 
channel. By using the moment generating functions (MGF) and Taylor series expansion, the exact and approximate closed-form expressions of ergodic capacity for DAS are derived, respectively. Besides, the outage capacity of the system is analyzed, and the corresponding outage probability is derived. As a result, an exact closed-form expression of outage probability is achieved. For a given outage probability, a practical iterative algorithm based on Newton's method for finding the outage capacity is proposed. Based on these theoretical expressions, the downlink capacity performance of DAS can be effectively evaluated. Moreover, the derived exact capacity expression avoids the error in the existing theoretical expression. Computer simulation shows that our exact expressions can match the simulation result very well, and the approximate expression is also close to the exact one.

The notations we use throughout this paper are as follows. Bold upper case letters denote matrices. $E\{\cdot\}$ denotes the expectation. The superscripts $(\cdot)^{T}$ and $(\cdot)^{H}$ are used to stand for the transpose and Hermitian transpose, respectively.

\section{System Model}

In this paper, we consider the distributed antenna systems with multiple receive antennas in a multicell environment. The main processing modules are centralized at a central unit and are connected with distributed antennas (DAs), and each of these DAs is also called an access point (AP). Through coaxial cables, fiber optics, or radio links, all the APs are connected with a home base station (BS) [3]. The structure of DAS can refer to Figure 1 in [6], where a cell is covered by a small BS and six APs, but each MS has $N$ receive antennas, which is different with [6] (only 1 receive antenna is considered in [6]). The total transmit power in one cell is assumed to be $P$, that is, $\sum_{m=0}^{6} P_{m}^{(k)}=P$, where $P_{m}^{(k)}$ is the transmit power of the $m$ th AP of the $k$ th cell, the small BS of each cell is indexed $m=0$, and home cell is indexed by $k=0$. Similar to [6], a single user scenario is considered here for analysis simplicity.

The transmitters from home BS and six APs and the receivers from MS construct a macroscopic MIMO fading channel, and channel matrix of the $k$ th cell can be expressed as

$$
\mathbf{H}^{(k)}=\left[\begin{array}{c}
\mathbf{h}_{1}^{(k)} \\
\vdots \\
\mathbf{h}_{N}^{(k)}
\end{array}\right]=\left[\begin{array}{cccc}
h_{1,0}^{(k)} \sqrt{L_{0}^{(k)}} & h_{1,1}^{(k)} \sqrt{L_{1}^{(k)}} & \cdots & h_{1,6}^{(k)} \sqrt{L_{6}^{(k)}} \\
\vdots & \vdots & \ddots & \vdots \\
h_{N, 0}^{(k)} \sqrt{L_{0}^{(k)}} & h_{N, 1}^{(k)} \sqrt{L_{1}^{(k)}} & \cdots & h_{N, 6}^{(k)} \sqrt{L_{6}^{(k)}}
\end{array}\right],
$$

where $\mathbf{h}_{n}^{(k)}=\left[h_{n, 0}^{(k)} \sqrt{L_{0}^{(k)}} \cdots h_{n, 6}^{(k)} \sqrt{L_{6}^{(k)}}\right], h_{n, m}^{(k)}$ denotes the short term fading from the $m$ th AP to the $n$th receive antenna in the $k$ th cell. For Rayleigh fading channel, $\left\{h_{n, m}^{(k)}\right\}$ are modeled as independent complex Gaussian random variables with zero-mean and unit variance. The channel is assumed to be static during a symbol duration. $L_{m}^{(k)}$ denotes the longterm fading corresponding to propagation pathloss from the $m$ th AP in the $k$ th cell, which can be expressed as $L_{m}^{(k)}=\left[d_{m}^{(k)}\right]^{-\beta}, \beta$ is the pathloss exponent, $d_{m}^{(k)}$ denotes the normalized distance between the mobile user and $m$ th AP in the $k$ th cell.

Let the transmitted signal vector be $\mathbf{s}^{(k)}=\left[s_{0}^{(k)}, \ldots, s_{6}^{(k)}\right]^{T}$, then, for the downlink, the received signal from the $n$th receive antenna of MS can be given by

$$
\begin{aligned}
r_{n} & =\mathbf{h}_{n}^{(0)} \mathbf{s}^{(0)}+\sum_{k=1}^{6} \sum_{m=0}^{6} h_{n, m}^{(k)} \sqrt{L_{m}^{(k)}} \boldsymbol{s}_{m}^{(k)}+w_{n} \\
& =D+I+w_{n}, \quad n=1,2, \ldots, N
\end{aligned}
$$

where $s_{m}^{(k)}$ denotes the transmitted signal from the $m$ th AP in the $k$ th cell with $E\left\{\left|s_{m}^{(k)}\right|^{2}\right\}=P_{m}^{(k)} . D=\mathbf{h}_{n}^{(0)} \mathbf{s}^{(0)}$ is the desired signal, $I$ is the interference signals from the other six cells. $w_{n}$ is the additive Gaussian noise for the $n$th receive antenna, and it is a complex Gaussian random variable with zero mean and variance $\sigma_{w}^{2}$. Because the number of the interference is sufficiently large and interfering sources are independent of each other, the interference plus noise is assumed to be a complex Gaussian random variable $z$ [6]. According to this, and using the Central Limit Theorem, the variance of the $z$ can be written as $\sigma_{z}^{2}=\sum_{k=1}^{6} \sum_{m=0}^{6} L_{m}^{(k)} P_{m}^{(k)}+\sigma_{w}^{2}$.

\section{Ergodic Capacity Analysis}

In this section, we will give the downlink capacity analysis of the DAS in a multicell environment. The ergodic capacity at a given location of the target mobile station can be expressed as

$$
C_{e}=E\{C\}=E\left\{\log _{2}(1+\rho)\right\}=\left(\frac{1}{\ln 2}\right) \int_{0}^{\infty} f(\rho) \ln (1+\rho) d \rho,
$$

where $C=\log _{2}(1+\rho)$ is the channel capacity and $f(\rho)$ is the probability density function (PDF) of $\rho$. The signal to interference plus noise ratio (SINR) $\rho$ is expressed as $\rho=$ $\sum_{n=1}^{N} \rho_{n}$ after MRC, where $\rho_{n}$ is the SINR of the $n$th receive antenna, it is an independent random variable for different $n$ and is given by [6]

$$
\rho_{n}=\frac{\mathbf{h}_{n}^{(0)} \mathbf{C}\left(\mathbf{h}_{n}^{(0)}\right)^{H}}{\sigma_{z}^{2}}=\frac{\sum_{m=0}^{6}\left|h_{n, m}^{(0)}\right|^{2} L_{m}^{(0)} P_{m}^{(0)}}{\sigma_{z}^{2}}
$$

where $\mathbf{C}$ is the covariance matrix of the transmitted vector $\mathbf{s}^{(0)}$ and can be given by a diagonal matrix $\operatorname{diag}\left(P_{0}^{(0)}, P_{1}^{(0)}\right.$, $\left.\ldots, P_{6}^{(0)}\right)$. With (4) and considering that $\left|h_{n, m}^{(k)}\right|^{2}$ is chi-square distributed with 2 degrees of freedom, the MGF of $\rho_{n}$ can be expressed as $M_{\rho_{n}}(s)=E\left\{e^{-s \rho_{n}}\right\}=\prod_{m=0}^{6}\left(1+s L_{m}^{(0)} P_{m}^{(0)} / \sigma_{z}^{2}\right)^{-1}$. 
Thus, utilizing the independence of $\left\{\rho_{n}\right\}$, the MGF of $\rho$ can be given by

$$
\begin{aligned}
M_{\rho}(s) & =E\left\{e^{-s \rho}\right\} \\
& =\prod_{n=1}^{N} E\left\{e^{-s \rho_{n}}\right\} \\
& =\prod_{n=1}^{N} M_{\rho_{n}}(s) \\
& =\prod_{m=0}^{6}\left(1+\frac{s L_{m}^{(0)} P_{m}^{(0)}}{\sigma_{z}^{2}}\right)^{-N} .
\end{aligned}
$$

According to [10], (5) can be further expressed as

$$
M_{\rho}(s)=\sum_{n=1}^{N} \sum_{m=0}^{6} w_{n, m}\left(s+a_{m}\right)^{-n}
$$

where $a_{m}=\sigma_{z}^{2} /\left(L_{m}^{(0)} P_{m}^{(0)}\right)$, and the coefficients $w_{n, m}$ are given by

$$
w_{n, m}=\left.\frac{1}{(N-n) !} \frac{d^{N-n}}{d s^{N-n}}\left[M_{\rho}(s)\left(s+a_{m}\right)^{N}\right]\right|_{s=-a_{m}} .
$$

Using (6) and the inverse Laplace transform, the PDF of $\rho, f(\rho)$ can be obtained as

$$
\begin{aligned}
f(\rho) & =\frac{\sum_{n=1}^{N} \sum_{m=0}^{6} w_{n, m} \rho^{n-1} \exp \left(-\rho a_{m}\right)}{\Gamma(n)} \\
& =\frac{\sum_{n=1}^{N} \sum_{m=0}^{6} w_{n, m} \rho^{n-1} \exp \left(-\sigma_{z}^{2} \rho /\left(L_{m}^{(0)} P_{m}^{(0)}\right)\right)}{\Gamma(n)} .
\end{aligned}
$$

When $N$ is equal to 1 , the coefficients $w_{n, m}$ in (7) are changed to

$$
w_{1, m}=\frac{\sigma_{z}^{2}}{L_{m}^{(0)} P_{m}^{(0)}} \prod_{j=0, \neq m}^{6} \frac{L_{m}^{(0)} P_{m}^{(0)}}{L_{m}^{(0)} P_{m}^{(0)}-L_{j}^{(0)} P_{j}^{(0)}}=\frac{\sigma_{z}^{2}}{L_{m}^{(0)} P_{m}^{(0)}} \pi_{m} .
$$

Thus, the corresponding PDF in (8) is reduced to (6) in [6]. This result shows that the PDF of $\rho$ for single receive antenna is a special case of $(8)$.

Substituting (8) into (3) gives

$$
\begin{aligned}
C_{e} & =\frac{\sum_{n=1}^{N} \sum_{m=0}^{6}\left(w_{n, m} / \ln 2\right) \int_{0}^{\infty} \ln (1+\rho) \rho^{n-1} \exp \left(-\sigma_{z}^{2} \rho / L_{m}^{(0)} P_{m}^{(0)}\right)}{\Gamma(n) d \rho} \\
& =\frac{\sum_{n=1}^{N} \sum_{m=0}^{6}\left(w_{n, m} a_{m}^{-n} / \ln 2\right) \int_{0}^{\infty} \ln (1+\rho)\left(a_{m}\right)^{n} \rho^{n-1} \exp \left(-a_{m} \rho\right)}{\Gamma(n) d \rho} .
\end{aligned}
$$

Utilizing the equality $d\left(-\sum_{l=0}^{n-1}\left(a_{m} \rho\right)^{l} e^{-a_{m} \rho} / l !\right) / d \rho=$ $\left(a_{m}\right)^{n} \rho^{n-1} e^{-a_{m} \rho} / \Gamma(n),(10)$ can be rewritten as

$C_{e}=\frac{\sum_{n=1}^{N} \sum_{m=0}^{6}\left(w_{n, m} a_{m}^{-n} / \ln 2\right) \int_{0}^{\infty}(1+\rho)^{-1} \sum_{l=0}^{n-1}\left(a_{m} \rho\right)^{l} e^{-a_{m} \rho}}{l ! d \rho}$.
Let $t=1+\rho,(11)$ can be further expressed as

$C_{e}$

$$
\begin{aligned}
& =\frac{(1 / \ln 2) \sum_{n=1}^{N} \sum_{m=0}^{6}\left(w_{n, m} a_{m}^{-n}\right) \sum_{l=0}^{n-1}\left(a_{m}\right)^{l} e^{a_{m} \int_{1}^{\infty} t^{-1}(t-1)^{l} e^{-a_{m} t}}}{l ! d t} \\
& =\frac{1}{\ln 2} \sum_{n=1}^{N} \sum_{m=0}^{6} w_{n, m} a_{m}^{-n} \sum_{l=0}^{n-1} \frac{e^{a_{m}}}{l !} \sum_{u=0}^{l}\left(\begin{array}{l}
l \\
u
\end{array}\right) \times\left(-a_{m}\right)^{l-u} \Gamma\left(u, a_{m}\right),
\end{aligned}
$$

where $\Gamma(\cdot, \cdot)$ is the incomplete Gamma function [10].

Using $\Gamma(u, v)=\Gamma(u) P_{u}(v)$ and $\Gamma(0, v)=E_{1}(v)[10],(12)$ can be simplified as

$$
\begin{aligned}
C_{e}= & \frac{1}{\ln 2} \sum_{n=1}^{N} \sum_{m=0}^{6} w_{n, m} a_{m}^{-n} \\
& \times\left\{\sum_{l=0}^{n-1} \frac{e^{a_{m}}}{l !}\left(-a_{m}\right)^{l} E_{1}\left(a_{m}\right)+\sum_{l=1}^{n-1} \sum_{u=1}^{l} \frac{e^{a_{m}}\left(-a_{m}\right)^{l-u}}{(l-u) !} \frac{P_{u}\left(a_{m}\right)}{u}\right\} \\
= & \frac{1}{\ln 2} \sum_{n=1}^{N} \sum_{m=0}^{6} w_{n, m} a_{m}^{-n} \\
& \times\left\{P_{n}\left(-a_{m}\right) E_{1}\left(a_{m}\right)+\frac{\sum_{u=1}^{n-1} P_{u}\left(a_{m}\right) P_{n-u}\left(-a_{m}\right)}{u}\right\} \\
= & \sum_{n=1}^{N} \sum_{m=0}^{6} \frac{w_{n, m}}{\ln 2}\left(\frac{L_{m}^{(0)} P_{m}^{(0)}}{\sigma_{z}^{2}}\right){ }^{n} \\
& \times\left\{P_{n}\left(-\frac{\sigma_{z}^{2}}{L_{m}^{(0)} P_{m}^{(0)}}\right) E_{1}\left(\frac{\sigma_{z}^{2}}{L_{m}^{(0)} P_{m}^{(0)}}\right)\right. \\
& \left.+\sum_{u=1}^{n-1} \frac{1}{u} P_{u}\left(\frac{\sigma_{z}^{2}}{L_{m}^{(0)} P_{m}^{(0)}}\right) P_{n-u}\left(-\frac{\sigma_{z}^{2}}{L_{m}^{(0)} P_{m}^{(0)}}\right)\right\}
\end{aligned}
$$

where $P_{n}(x)=\sum_{l=0}^{n-1} x^{l} e^{-x} / l$ ! is the Poisson distribution and $E_{1}(\cdot)$ denotes the first-order exponential integral function. Equation (13) is an exact closed-form expression of ergodic capacity of DAS in the multicell environment. It is shown that this theoretical formula will have good agreement with simulation result. When $N$ is equal to 1 , using (9), (12) is reduced to

$$
\begin{aligned}
C_{e} & =\frac{1}{\ln 2} \sum_{m=0}^{6}\left(w_{1, m} a_{m}^{-1}\right) e^{a_{m}} E_{1}\left(a_{m}\right) \\
& =-\frac{1}{\ln 2} \sum_{m=0}^{6} \pi_{m} \exp \left(\frac{\sigma_{z}^{2}}{L_{m}^{(0)} P_{m}^{(0)}}\right) E_{i}\left(-\frac{\sigma_{z}^{2}}{L_{m}^{(0)} P_{m}^{(0)}}\right),
\end{aligned}
$$

where $E_{i}(x)$ is the exponential integral function and $E_{1}(x)=$ $-E_{i}(-x)$. This is an exact capacity expression for single receive antenna case, it has almost the same form as (7) in [6]. However, [6, equation (7)] has minor error in exponential term, and thus it cannot match the simulation results well. 
To further simplify the calculation in (13), we will give a simple approximate capacity expression. We may use Taylor series expansion at $\rho=\rho_{0}=E\{\rho\}$ to approximate $\ln (1+\rho)$ as

$$
\begin{aligned}
\ln (1+\rho) \cong & \ln \left(1+\rho_{0}\right)+\left(1+\rho_{0}\right)^{-1}\left(\rho-\rho_{0}\right) \\
& -0.5\left(1+\rho_{0}\right)^{-2}\left(\rho-\rho_{0}\right)^{2} .
\end{aligned}
$$

Substituting (15) into (3) yields the approximate capacity as

$$
\begin{gathered}
C_{a} \cong \frac{1}{\ln 2} E\left\{\ln \left(1+\rho_{0}\right)+\left(1+\rho_{0}\right)^{-1}\left(\rho-\rho_{0}\right)\right. \\
\left.-0.5\left(1+\rho_{0}\right)^{-2}\left(\rho-\rho_{0}\right)^{2}\right\} \\
=\frac{1}{\ln 2}\left[\ln \left(1+\rho_{0}\right)-0.5\left(1+\rho_{0}\right)^{-2}\left(E\left\{\rho^{2}\right\}-\rho_{0}^{2}\right)\right],
\end{gathered}
$$

where

$$
\begin{aligned}
\rho_{0} & =E\{\rho\} \\
& =\int_{0}^{\infty} \rho f(\rho) d \rho \\
& =\sum_{n=1}^{N} \sum_{m=0}^{6} w_{n, m}\left(\frac{L_{m}^{(0)} P_{m}^{(0)}}{\sigma_{z}^{2}}\right)^{n+1}\left[\frac{\Gamma(n+1)}{\Gamma(n)}\right], \\
E\left\{\rho^{2}\right\} & =\int_{0}^{\infty} \rho^{2} f(\rho) d \rho \\
& =\sum_{n=1}^{N} \sum_{m=0}^{6} w_{n, m}\left(\frac{L_{m}^{(0)} P_{m}^{(0)}}{\sigma_{z}^{2}}\right)^{n+2}\left[\frac{\Gamma(n+2)}{\Gamma(n)}\right] .
\end{aligned}
$$

Equation (16) is an approximate closed-form expression of ergodic capacity of DAS in the multicell environment. Compared to the exact expression (13), it has simpler form. Moreover, this approximate value will be shown to have almost the same result as the exact value.

\section{Outage Capacity Analysis}

In this section, we will give the outage capacity analysis of the DAS in a multicell environment and derive the corresponding outage probability. Since the channel capacity is a random variable, it is meaningful to consider its statistical distribution. A useful measure of statistical characteristic is the outage capacity [11]. For the given outage capacity $C_{o}$, $P\left(C_{o}\right)$ is defined as the probability that the channel capacity $C$ falls below $C_{o}$. Thus, the outage capacity probability at a given location of the target mobile station can be expressed as

$P\left(C_{o}\right)=\operatorname{Prob}\left(C \leq C_{o}\right)=\operatorname{Prob}\left(\rho \leq 2^{C_{o}}-1\right)=\int_{0}^{2^{C_{o}}-1} f(\rho) d \rho$.
Substituting (8) into (18) yields

$$
\begin{aligned}
P\left(C_{o}\right) & =\frac{\int_{0}^{2^{C_{o}}-1} \sum_{n=1}^{N} \sum_{m=0}^{6} w_{n, m} \rho^{n-1} \exp \left(-\sigma_{z}^{2} \rho /\left(L_{m}^{(0)} P_{m}^{(0)}\right)\right)}{\Gamma(n) d \rho} \\
& =\frac{1-\sum_{n=1}^{N} \sum_{m=0}^{6} w_{n, m} \int_{2}^{\infty} C_{o-1} \rho^{n-1} \exp \left(-\sigma_{z}^{2} \rho /\left(L_{m}^{(0)} P_{m}^{(0)}\right)\right)}{\Gamma(n) d \rho} .
\end{aligned}
$$

Using the incomplete Gamma function $\Gamma(\mu, v) \triangleq$ $\int_{v}^{+\infty} t^{\mu-1} e^{-t} d t[10]$, (19) can be further expressed as

$$
P\left(C_{o}\right)=1-\sum_{n=1}^{N} \sum_{m=0}^{6} \frac{w_{n, m}}{\Gamma(n)}\left(\frac{L_{m}^{(0)} P_{m}^{(0)}}{\sigma_{z}^{2}}\right)^{n} \Gamma\left(n, \frac{\left(2^{C_{o}}-1\right) \sigma_{z}^{2}}{L_{m}^{(0)} P_{m}^{(0)}}\right) .
$$

This is an exact closed-form expression of outage probability of DAS in the multicell environment, which is shown to match the simulation well. For a given outage probability $\alpha=P\left(C_{o}\right)$, we propose a practical iterative algorithm based on Newton's method for finding the outage capacity $C_{\alpha}$.

In the following, we express solving the outage capacity from (20) as finding the root of $F\left(C_{\alpha}\right)=0$ with

$$
\begin{aligned}
F\left(C_{\alpha}\right)= & 1-\sum_{n=1}^{N} \sum_{m=0}^{6} \frac{w_{n, m}}{\Gamma(n)}\left(\frac{L_{m}^{(0)} P_{m}^{(0)}}{\sigma_{z}^{2}}\right)^{n} \\
& \times \Gamma\left(n, \frac{\left(2^{C_{o}}-1\right) \sigma_{z}^{2}}{L_{m}^{(0)} P_{m}^{(0)}}\right)-\alpha,
\end{aligned}
$$

where $\alpha$ is the given outage probability. We now show that $F\left(C_{\alpha}\right)$ is a monotonic function with $F^{\prime}\left(C_{\alpha}\right)>0$. Differentiating $F\left(C_{\alpha}\right)$ with respect to $C_{\alpha}$ gives

$$
\begin{aligned}
F^{\prime}\left(C_{\alpha}\right)= & \frac{\partial F\left(C_{\alpha}\right)}{\partial C_{\alpha}} \\
= & f\left(2^{C_{\alpha}}-1\right) 2^{C_{\alpha}} \ln 2 \\
= & \sum_{n=1}^{N} \sum_{m=0}^{6} \frac{w_{n, m}}{\Gamma(n)}\left(2^{C_{\alpha}}-1\right)^{n-1} \exp \left(-\frac{\left(2^{C_{\alpha}}-1\right) \sigma_{z}^{2}}{L_{m}^{(0)} P_{m}^{(0)}}\right) \\
& \times 2^{C_{\alpha}} \ln 2>0 .
\end{aligned}
$$

The derivative $F^{\prime}\left(C_{\alpha}\right)$ is positive in (22). Thus, $F\left(C_{\alpha}\right)$ is a strictly monotonically increasing function of $C_{\alpha}$. From (21) and (19), we can obtain

$$
\begin{gathered}
F(\infty)=\lim _{C_{\alpha} \rightarrow \infty} F\left(C_{\alpha}\right)=1-\alpha>0, \\
F(0)=-\alpha<0 .
\end{gathered}
$$

This is because outage probability $\alpha \in(0,1)$. Based on the above analysis, the equation $F\left(C_{\alpha}\right)=0$ is shown to have a unique solution for $C_{\alpha}>0$.

There are many methods such as bisection for finding the root of a strictly monotonic function. We propose to 
use Newton's method to find the root iteratively because it has the quadratic convergence rate. Newton's method is described as follows:

$$
C_{\alpha, n+1}=C_{\alpha, n}-\frac{F\left(C_{\alpha, n}\right)}{F^{\prime}\left(C_{\alpha, n}\right)},
$$

where $C_{\alpha, n}$ is the outage capacity value at the $n$th iteration. $F\left(C_{\alpha, n}\right)$ and $F^{\prime}\left(C_{\alpha, n}\right)$ are computed by (21) and (22), respectively.

In addition, the proposed analysis method for the downlink capacity can be easily extended to the uplink case by the appropriate transformation and mathematical calculation, and correspondingly, closed-form expressions of ergodic capacity and outage probability of DAS uplink can be derived, respectively. Thus, some similar results can be obtained. Here, we no longer give specific details due to much more similarities.

\section{Simulation Results and Theoretical Evaluation}

Simulation results are provided to evaluate the effectiveness of the derived capacity expressions. We set the pathloss exponent $\beta=4$ and the total power to noise ratio $P / \sigma_{n}^{2}=$ $15 \mathrm{~dB}$. The transmit power of each AP is $0.1 P$, and the transmit power of the home BS is $0.4 P$ in DAS. The MonteCarlo method is employed for simulation. The exact and approximate theoretical values of capacity are from (13) and (16), respectively. The normalized distance from the home BS to the direction of the worst position $\mathrm{W}$ (this position can refer to Figure 1 in [6]) on the cell boundary is indexed by $d(0 \leq d \leq 1)$. " $v \mathrm{Rx}$ " denotes the system with $v$ receive antenna. Three theoretical capacities are considered in the comparison. The ergodic capacity described by (13) is referred to as "theory 1 ," the one described by $[6$, equation (7)] is referred to as "theory 2," and the one described by (16) is referred to as "theory 3."

Figure 1 shows the ergodic capacity versus the normalized distance from home BS $d$. The derived "theory 1" and the existing "theory 2" [6] are compared. As shown in Figure 1, the theoretical analysis from "theory 1" is in good agreement with the simulation results, whereas the theoretical evaluation from "theory 2" is different with the simulation results. This is because the "theory 2" in [6] has error. It is found that the systems with $2 \mathrm{Rx}$ outperform those with $1 \mathrm{Rx}$ because the former has greater diversity than the latter. From Figure 1, we can see nonmonotonic relationship between the capacity and the normalized distance $d$. Namely, when the MS is far away from the home BS and moves around $d=0.4$, the capacity is decreasing since the received signal quality of MS starts to become worse. Whereas when the MS moves from $d=0.4$ to $d=0.6$, the signal from an AP becomes dominant, and the corresponding capacity is increasing. However, when MS moves from $d=0.6$ to $d=1$, the capacity is decreasing because the interference signal from neighbor cell starts to become strong. The above results show that the derived capacity expression of DAS is effective.

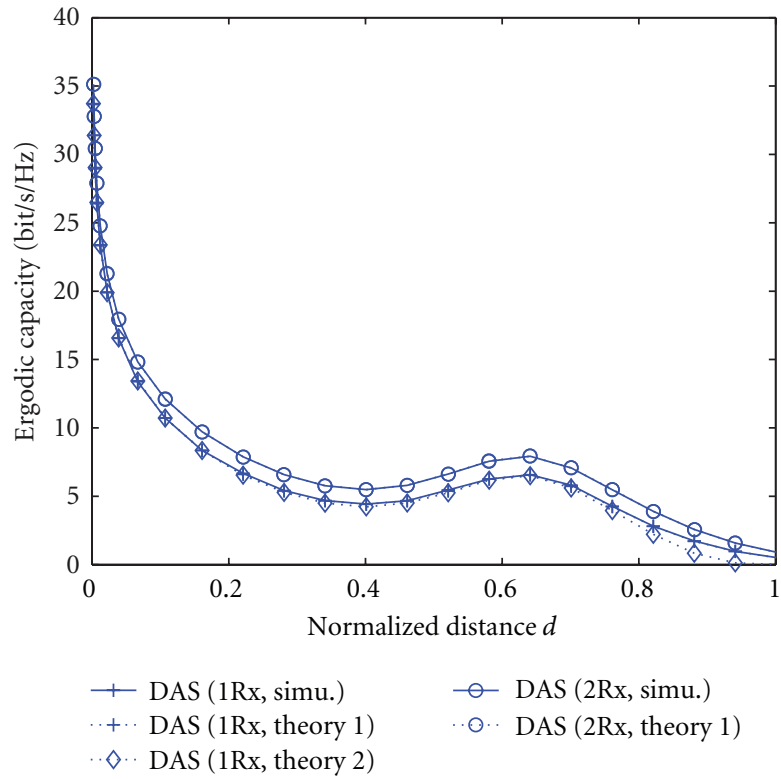

FIGURE 1: Ergodic capacity versus the normalized distance from the home BS.

In Figure 2, the approximate performance formula (theory 3 ) is compared with the accurate performance formula (theory 1). The number of receive antenna is set as to be 1,2 , and 3. It is found that "theory 3 " has the values very close to that of "theory 1." Moreover, the calculation of the former is relatively simpler than that of the latter. Thus, this approximate expression can be used for the performance evaluation of DAS. Besides, as the number of receive antennas increases, the performance of the two theoretical formulae is both effectively improved due to the greater diversity. The above results show that the derived approximate expression of ergodic capacity is also valid.

In Figure 3, we plot the theoretical outage probability and corresponding simulation of DAS with different receive antennas. The outage capacity $C_{o}$ is set as to $6 \mathrm{bit} / \mathrm{s} / \mathrm{Hz}$. The theoretical outage probability is evaluated by (20). Computer simulation results verify the accuracy of the derived theoretical formula. It is observed that the outage probability of DAS with three receive antennas is lower than that with two receive antennas because the former has greater diversity than the latter. For the same reason, the DAS with two receive antennas has lower outage probability than that with one receive antenna. Besides, some results similar to Figure 1 can be found, that is, we can observe nonmonotonic relationship between the outage probability and the normalized distance $d$. Specifically, the outage probability firstly increases when the MS is far away from the home BS, and then the outage probability decreases when the MS moves to $d=0.6$. Finally, the outage probability increases again when the MS moves to the cell boundary. This is because the signal from an AP becomes dominant near $d=0.6$. The above result shows the derived outage probability is also effective for the performance evaluation. 


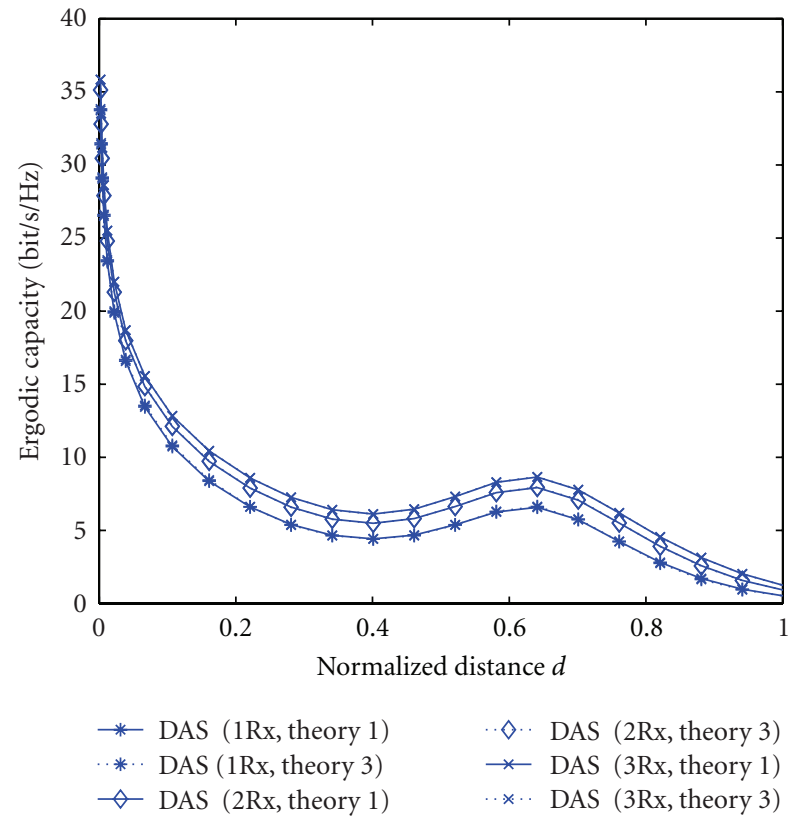

Figure 2: Comparison between exact and approximate ergodic capacity.

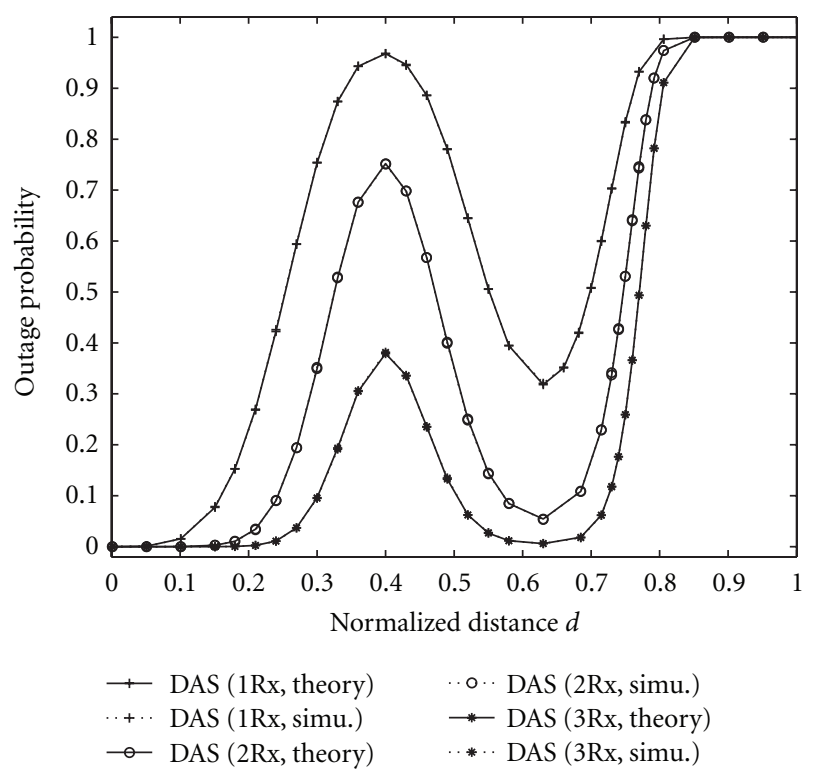

Figure 3: Outage probability versus the normalized distance from the home BS.

Figure 4 shows the outage capacity versus the normalized distance from home BS $d$. The number of receive antenna $N=1,2$, and 3, the outage probability is set as to 0.05 . The outage capacity is calculated by (24). From this figure, we can also see nonmonotonic relationship between the outage capacity and the normalized distance $d$. The reason can refer to the analysis in Figure 1. Moreover, the larger the value of $\mathrm{N}$, the bigger is the outage capacity as expected. Comparing the results of Figures 4 and 2, we can find that the outage

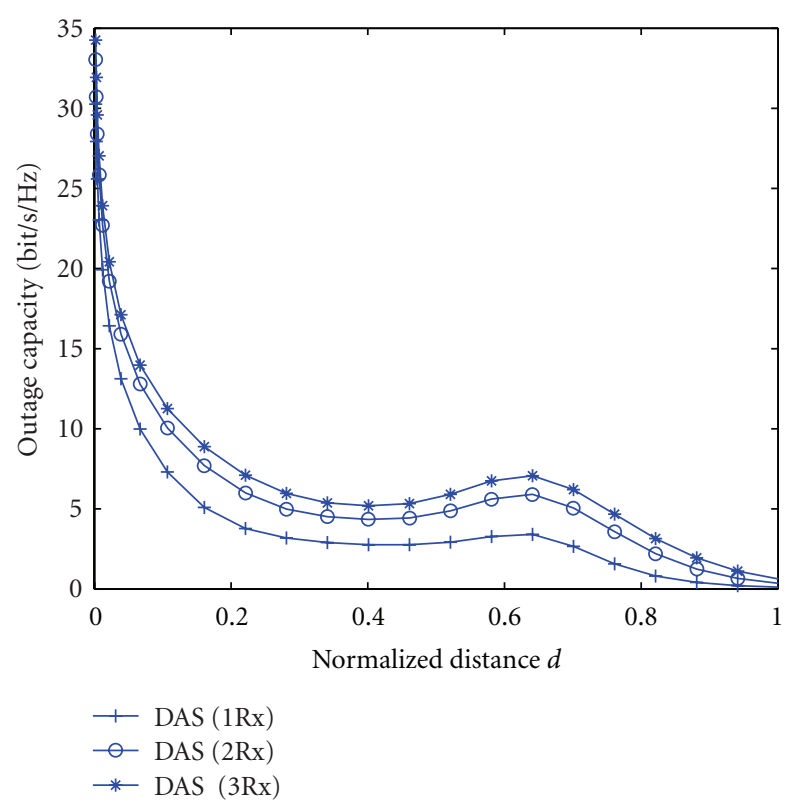

FIGURE 4: Outage capacity versus the normalized distance from the home BS.

capacity is lower than the corresponding ergodic capacity due to the limitation of the outage probability.

\section{Conclusions}

We have studied the downlink performance and capacity of DAS in MIMO fading and multicell environment. The MGF and PDF of SINR of the system are derived. Based on these results, exact and approximate closed-form expressions of the ergodic capacity of DAS are obtained, respectively. The exact expressions include the existing expression with single receive antenna as a special case and correct minor error of the latter. Moreover, according to the outage capacity analysis of the system, an exact closed-form expression of outage probability is also derived. Newton's method is proposed to find the outage capacity value for a given outage probability. Computer simulation shows that the derived theoretical expressions can match the simulation results well, and the application of multiple receive antennas can obviously increase the system capacity. Thus, these expressions provide good performance evaluation for DAS in the multicell environment and avoid the conventional need for numerical integration or Monte Carlo simulation.

\section{Acknowledgments}

The authors would like to thank for the anonymous reviewer and Associate Editor for their valuable comments and suggestions, which greatly improve the quality of this paper. The work was supported in part by National Natural Science Foundation of China (61172077), Doctoral Fund of Ministry of Education of China (20093218120021), Fundamental Research Funds for the Central Universities (NS2012075), National Key Project of Chinese Ministry of Science and 
Technology (2009ZX03003-006-03), and Shanghai Municipal Natural Science Foundation (10ZR1436000).

\section{References}

[1] X. Chen, Z. Zhang, and H. H. Chen, "On distributed antenna systems with limited feedback precoding: opportunities and challenges," IEEE Wireless Communications, vol. 17, no. 2, pp. 80-88, 2010.

[2] S. Zhou, M. Zhao, X. Xu, J. Wang, and Y. Yao, "Distributed wireless communication system: a new architecture for future public wireless access," IEEE Communications Magazine, vol. 41, no. 3, pp. 108-113, 2003.

[3] H. M. Chen, J. B. Wang, and M. Chen, "Letter transmission systems outage performance of distributed antenna systems over shadowed nakagami-m fading channels," European Transactions on Telecommunications, vol. 20, no. 5, pp. 531535, 2009.

[4] L. Dai, S. Zhou, and Y. Yao, "Capacity analysis in CDMA distributed antenna systems," IEEE Transactions on Wireless Communications, vol. 4, no. 6, pp. 2613-2620, 2005.

[5] Z. Feng, Z. Jiang, W. Pan, and D. Wang, "Capacity analysis of generalized distributed antenna systems using approximation distributions," in Proceedings of the 11th IEEE Singapore International Conference on Communication Systems (ICCS '08), pp. 828-830, November 2008.

[6] W. Choi and J. G. Andrews, "Downlink performance and capacity of distributed antenna systems in a multicell environment," IEEE Transactions on Wireless Communications, vol. 6, no. 1, pp. 69-73, 2007.

[7] J. Park, E. Song, and W. Sung, "Capacity analysis for distributed antenna systems using cooperative transmission schemes in fading channels," IEEE Transactions on Wireless Communications, vol. 8, no. 2, pp. 586-592, 2009.

[8] X. Li, M. Luo, M. Zhao, L. Huang, and Y. Yao, "Downlink performance and capacity of distributed antenna system in multi-user scenario," in Proceedings of the 5th International Conference on Wireless Communications, Networking and Mobile Computing (WiCOM '09), pp. 1-4, September 2009.

[9] H. M. Chen and M. Chen, "Capacity of the distributed antenna systems over shadowed fading channels," in Proceedings of the IEEE 69th Vehicular Technology Conference (VTC'09), pp. 1-4, April 2009.

[10] I. S. Gradshteyn and I. M. Ryzhik, Table of Integrals, Series, and Products, Academic, San Diego, Calif, USA, 7th edition, 2007.

[11] E. G. Larsson and P. Stoica, Space-Time Block Coding for Wireless Communications, Cambridge University Press, Cambridge, UK, 2003. 

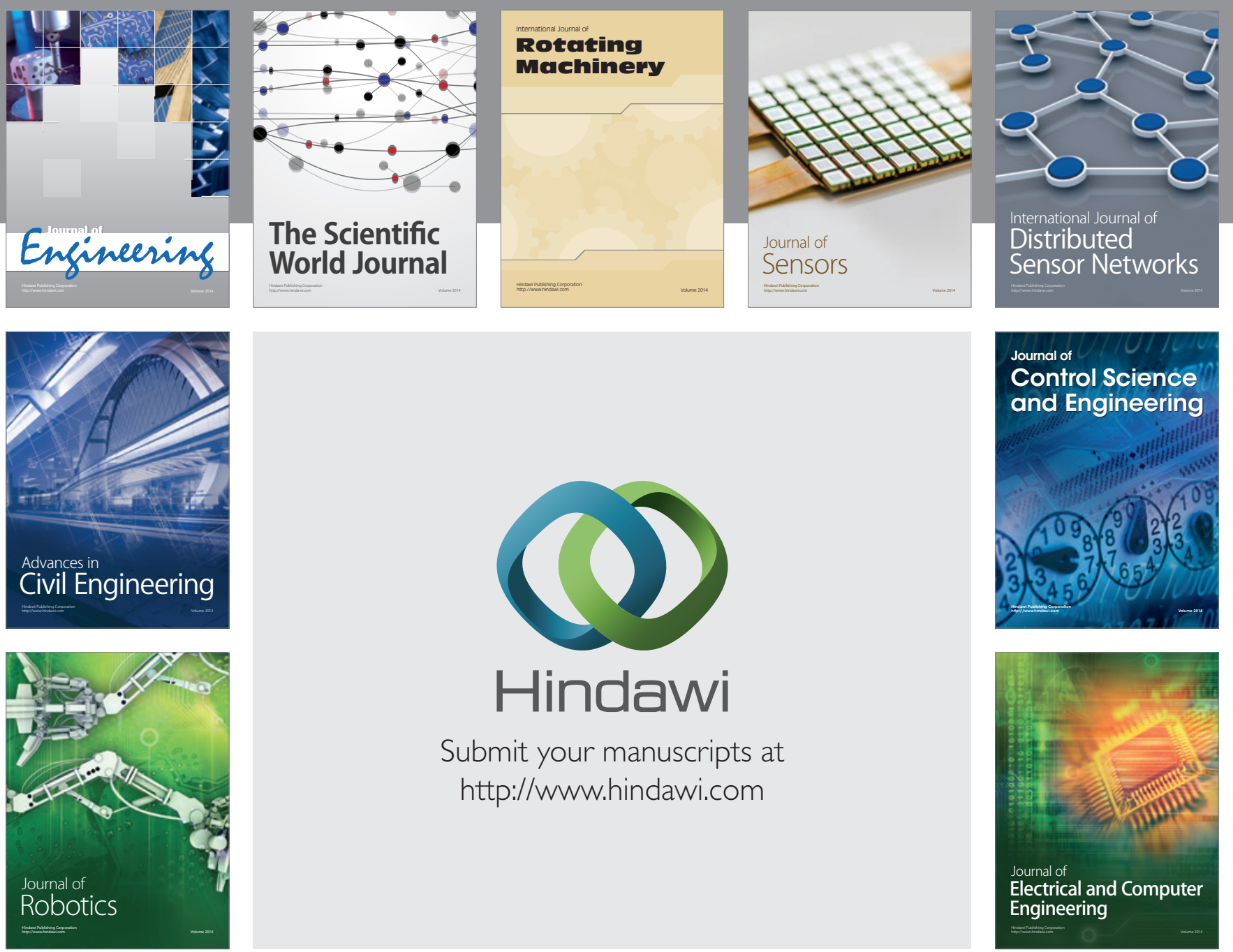

Submit your manuscripts at

http://www.hindawi.com
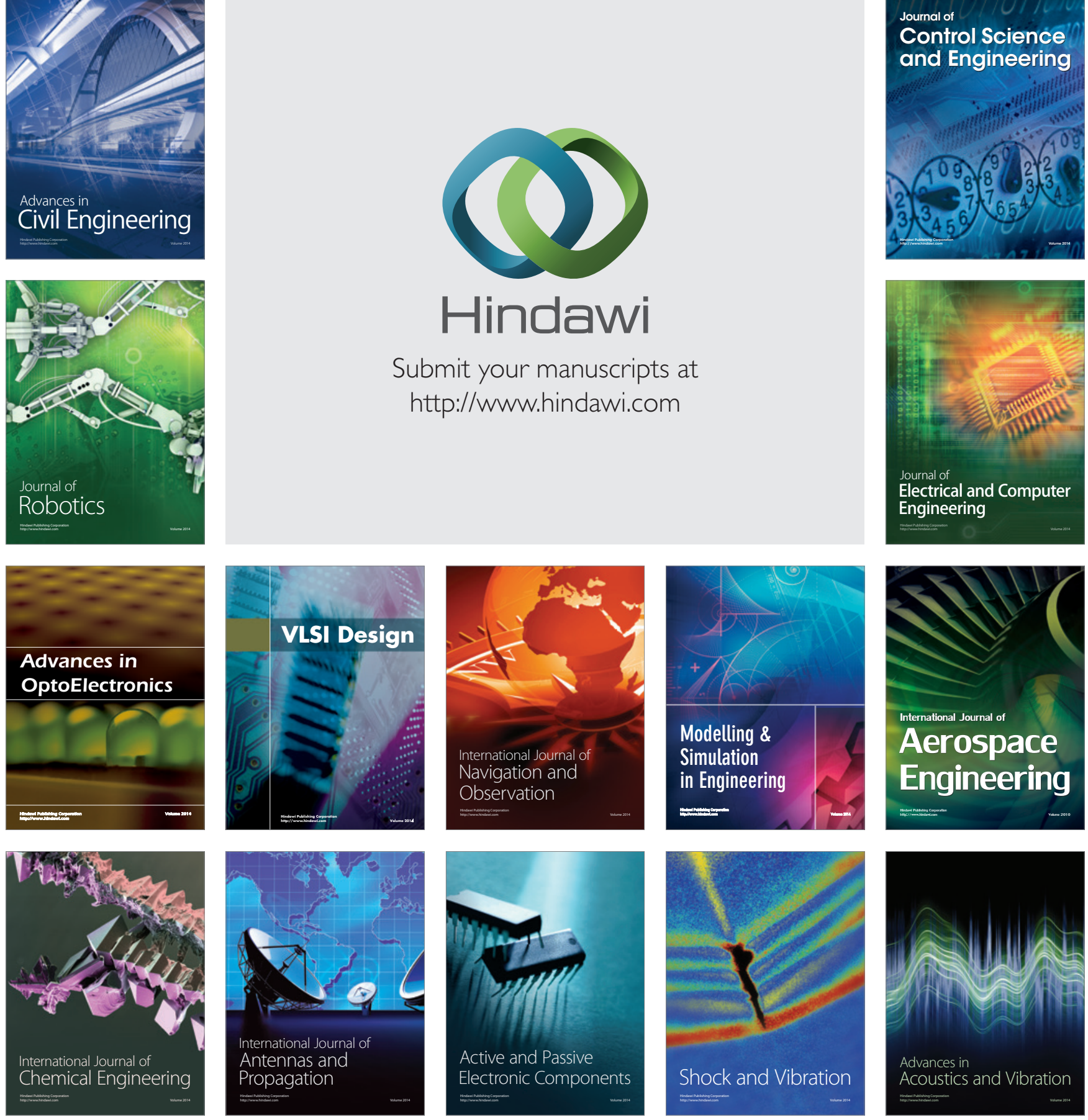